\title{
INTER-RELAÇÃO DE CONTEÚDOS NA CONSTRUÇÃO DO CONHECIMENTO POR CONFLITO COGNITIVOO
}

\author{
Selma de Cássia Martinelli*
}

Fermino Fernandes Sisto

\begin{abstract}
RESUMO. O trabalho estuda a problemática da transferência, em situação experimental, de aprendizagem por conflito cognitivo, abordando o aspecto maturacional, o gênero e a influência de diferentes sequiências de aprendizagem de conteúdos. Foram formados aleatoriamente quatro grupos (16;21; 20 e 18 sujeitos), homogêneos em relação à idade. Os sujeitos foram submetidos a sessões de intervenção nos conteúdos de formas parcialmente escondidas (FPE) e de eqüidistância (EQ), por intermédio da técnica do conflito cognitivo. Um grupo recebeu intervenção em FPE; outro em EQ; o terceiro grupo em FPE e depois em EQ; e o quarto em EQ e depois em FPE. Foram realizadas três sessões individuais com duração média de 30 minutos. Todos os sujeitos passaram por pós-testes, imediatamente ao final da última intervenção e trinta dias após o primeiro pós-teste, em três provas, quais sejam: formas parcialmente escondidas, eqüidistância e posições possíveis dos dados, em sequiências aleatórias. Três aspectos devem ser ressaltados, dos resultados obtidos nos experimentos: a maturação e o gênero mostraram exercer um papel na aprendizagem; houve mudanças no desempenho dos sujeitos em relação a um conteúdo não ensinado, sugerindo a ocorrência de transferência; houve superioridade de uma situação experimental sobre a outra.
\end{abstract}

Palavras-chaves: conflito cognitivo, transferência de aprendizagem, maturação.

\section{THE INTERRELATION OF CONTENTS IN THE KNOWLEDGE CONSTRUCTION BY COGNITIVE CONFLICT}

\begin{abstract}
The transference issue, maturity, gender and different sequences of learning were studied in experimental learning contexts mediated by cognitive conflict. Four groups (16;21;20 and 18 subjects) randomly composed were submitted to three thirty minutes intervention sessions using the contents "Shapes partially hidden" and "Equidistance". "Shapes partially hidden" was presented to the first group; "Equidistance" to the second; "Shapes partially hidden" followed by "Equidistance" to the third; and "Equidistance" followed by "Shapes partially hidden" to the last one. All subjects were post tested immediately after the last session and thirty days after the first post test, by three tasks, "Shapes partially hidden", "Equidistance" and "Dice positions", randomly assigned. Three aspects were highlighted as result of the experiments: the maturity and gender seems to interact with learning; there were differences among the groups in relation to non taught contents suggesting an effect of transference; and the groups were differentiated by their achievements.
\end{abstract}

Key words: cognitive conflict, transference of learning, maturity.

É aceito o fato de o ser humano estar imerso num meio em que a aprendizagem ocorre de forma constante. A todo instante nos deparamos com situações novas ou que, mesmo conhecidas, comportam alguns elementos que não estavam presentes ou que se apresentam diferentemente na nova situação.

Por diferentes perspectivas teóricas tem-se procurado investigar a ocorrência de aprendizagens, entre as quais, pesquisas baseadas no modelo teórico piagetiano. Modelos experimentais em que o conflito cognitivo é introduzido para provocar mudanças nas bases conceituais dos sujeitos têm sido estudados em diversas partes do mundo.

Ainda na perspectiva do uso do conflito, alguns pesquisadores têm-se detido nas investigações que procuram verificar a transferência de aprendizagem, apontada como uma das prerrogativas mais frutíferas. Espera-se que o aprendiz seja capaz de utilizar as novas informações adquiridas em diferentes

\footnotetext{
Professora doutora do Departamento de Psicologia Educacional da Unicamp.

\# Professor doutor da Universidade São Francisco.

Endereço para correspondência: Rua Carlos Guimaraes, 150. Ap. 82, CEP 13024-200, Campinas-São Paulo.
} 
circunstâncias e situações. Espera-se, assim, que o aprendido possa ser evocado, não somente na situação em que foi gerado, mas em diferentes contextos da vida. Por isso, estudar como se dá a ocorrência da transferência e que situações podem favorecê-la tem despertado o interesse de pesquisadores envolvidos com a aprendizagem. Buscar a possibilidade dessa ocorrência dentro do modelo teórico construtivista, em que Piaget apregoou ser o conhecimento construído conteúdo por conteúdo, também tornou-se objeto de investigação. Já no final dos anos 50 (Morf, 1959) e início da década de 60, com os estudos de Gruen (1965) e Brison (1966), apontava-se para as dificuldades de se obter a transferência de aprendizagem. Nas décadas seguintes, Waghorn \& Sullivan (1970), Padilla \& Ollila (1980) recolocam em suas pesquisas essa mesma preocupação, demonstrando mais uma vez não se ter consenso quanto à possibilidade de obtenção de transferência. Esses primeiros estudos deixaram em aberto essa questão que, do ponto de vista do ensino, é uma das mais importantes.

A retomada dessa temática ocorre na década de 90, com os estudos sobre aprendizagem dos possíveis ou da criação das novidades na perspectiva de Piaget (1985). Esses novos estudos de aprendizagem realizados, ora analisaram a transferência para um conteúdo operatório (Louro, 1993;Yaegashi, 1994; Pereira, 1995; e Liesenberg, 1994), ora para um conteúdo sobre a formação de possíveis (Martinelli, 1994; Pavanello, 1995). Todos tinham em comum o fato de terem usado o conflito cognitivo como técnica para intervenção. Dos seis estudos citados que procuraram verificar a transferência, apenas dois (Louro, 1993; Yaegashi, 1994) encontraram resultados que permitiram verificar essa ocorrência, sendo que no primeiro estudo, a aprendizagem se deu num conteúdo de possível e a transferência foi verificada em conservação de massa (conteúdo operatório). No segundo estudo, verificou-se a situação inversa, ou seja, aprendizagem em conteúdo operatório de inclusão de classes e transferência para outros conteúdos sobre a formação de possíveis.

Os resultados desses estudos mostram-se, mais uma vez, lacunares para responder à questão da transferência em aprendizagem. Também acabaram por chamar a atenção para o fato de que outros fatores podem estar colocados na explicação desse processo. Nesse contexto, as influências do gênero e da maturação nas aprendizagens têm despertado o interesse de pesquisadores, fazendo-os, cada vez mais, descobrir os meandros desse processo e as variáveis que podem interferir nele. Na literatura encontram-se alguns estudos realizados que buscam relações entre o gênero e a aprendizagem, entre eles Riding \& Armstrong (1982) e Toh (1993). Os resultados apontaram a tendência de as meninas se saírem melhor que os meninos na memorização de fatos e obterem melhor desempenho em habilidades práticas.

Em crianças em idade pré-escolar as relações entre gênero e aprendizagem referem-se basicamente aos aspectos da percepção, memória, estilos cognitivos, pensamento, aprendizagem de conceitos temporais e espaciais e resolução de problemas (por exemplo, Worthington \& Typo, 1980; Lutzer, 1987). Os resultados desses estudos mostraram-se contraditórios e incipientes, apontando a necessidade de outras investigações.

Os aspectos maturacionais também têm despertado a atenção de alguns pesquisadores. Para Piaget (1964) a maturação é um dos quatro elementos envolvidos no processo de desenvolvimento e na aprendizagem, e seria uma continuação da embriogênese. No entanto, apesar de ser uma condição necessária, ela não é suficiente para o aparecimento de certas condutas, pois o exercício funcional e a experiência (física e social) também se impõem nesse processo. Martinelli (1999) também verificou a influência da idade em crianças submetidas a situações de aprendizagem, sendo que as mais velhas sempre apresentaram um desempenho superior ao das mais novas.

Garcia (1999) procurou investigar tanto a influência da maturação quanto a do gênero na aprendizagem, provocada por conflito sociocognitivo em conservação de comprimento, e as possibilidades de completamento de uma figura apresentada de forma parcial. Os resultados mostraram que os meninos aprenderam mais que as meninas na conservação de comprimento, enquanto as meninas se saíram melhor na construção de novas formas para o objeto escondido. Em ambos os conteúdos houve superioridade no desempenho das crianças mais velhas, quando comparadas às mais novas.

A tendência verificada nesses estudos, de que o gênero e a maturação poderiam ser fatores a mais na explicação da aprendizagem, parece ter norteado o estudo realizado por Sisto (2000), que vem recolocar a possibilidade de ocorrência do processo de transferência em aprendizagem. Em seu experimento procurou provocar aprendizagem em conservação de massa e verificar a possibilidade de transferência para a conservação de líquido, estudando até que ponto as variáveis idade, tipo de intervenção (conflito puramente verbal e conflito verbal com algumas situações de demonstração) e resultados imediatos e 
postergados da intervenção interfeririam no processo de transferência. Os resultados obtidos no experimento sugerem que a idade influi na transferência. $O$ quantum de aprendizagem verificado através de dois pós-testes teve ação apenas imediata e as técnicas de conflito cognitivo não produziram diferenças.

Assim, considerando essas novas indicações da literatura é que o presente estudo, ao propor um experimento de aprendizagem, segundo o enfoque teórico psicogenético, procura trazer novos elementos para se pensar a problemática da transferência. Além de abordar o aspecto maturacional e o do gênero, também procurou analisar a influência de diferentes procedimentos de intervenção, caracterizados pela sequiência dos conteúdos trabalhados na aprendizagem.

\section{MÉTODO}

\section{Objetivos}

Foram colocados dois objetivos neste estudo: por um lado, verificar se há um conteúdo ou uma seqüência de conteúdos que mais favoreça a construção de outro, não utilizado na intervenção, mas que solicite o mesmo mecanismo psicológico da analogia; e por outro lado, verificar se a idade e o gênero interferem na aprendizagem dos grupos experimentais submetidos a diferentes tipos de intervenção.

\section{Hipóteses}

Duas hipóteses foram levantadas. A primeira afirma que a "seqüência de conteúdos utilizada na intervenção se reflete diferentemente na elaboração de um novo conteúdo"; e a segunda, que "a idade e o gênero dos sujeitos influenciam o processo de aprendizagem".

\section{Sujeitos experimentais}

Os sujeitos foram sorteados aleatoriamente para formar quatro grupos, divididos conforme os tipos de intervenção, descritos mais adiante. $\mathrm{O}$ experimento foi finalizado com 16;21; 20 e 18 sujeitos respectivamente, em um total de 75 sujeitos. As médias de idade dos grupos, em meses, foram respectivamente: 67,44; 70,29; 70 e 67,28. Adotandose o nível de significância de 0,05 para verificar a homogeneidade dos grupos em relação à idade, encontrou-se um valor de $\mathrm{F}=1,198$ e um $\mathrm{p}=0,317$, o que permitiu considerar os grupos como homogêneos ou provenientes da mesma população.

\section{Descrição das provas e critérios de classificação}

As provas, materiais, forma de aplicação e critérios de avaliação são praticamente os mesmos propostos por Piaget (1985).

Para a aplicação da prova da Construção de Eqüidistâncias, os materiais usados foram casas de cores diferentes e uma árvore, carrinhos e sinais de trânsito, animais e bonecos, todos em miniatura. A experimentadora solicitava que a criança colocasse as casas a uma distância igual da árvore, e depois colocasse de outros jeitos, mas sempre mantendo a mesma distância entre as casas e a árvore. As casas, os carrinhos, os animais ou bonecos eram oferecidos gradualmente, até o máximo de doze miniaturas.

Critérios de classificação: Nível IA - a criança procede a uma reunião das casas através de um alinhamento vertical ou horizontal, figuras em curva, ziguezague, casas em desordem, amontoadas, entre outras maneiras. Nível IB - ocorre a predominância das condutas do nível anterior acrescidas de outras como: casas ao redor da árvore mas colocadas desordenadamente, círculo com árvore colocada do lado de fora, vários alinhamentos colocados sem simetria, além de formas quadradas, retangulares, triangulares, que podem ser abertas ou fechadas. Nível II - ocorre o predomínio de figuras que envolvem o ponto central, como o quadrado e o triângulo, entre outras, fazendo correções na tentativa de chegar à eqüidistância. Nível III - as únicas construções aceitas são o círculo ou o semicírculo, que podem apresentar variações em suas dimensões.

Formas Possíveis de uma Realidade Parcialmente Escondida: nesta prova, o objetivo era verificar como as crianças procediam diante de um objeto parcialmente escondido, imaginando várias alternativas para a parte invisível. O material foram objetos com formas regulares e irregulares. A criança teve à sua disposição papel e lápis para que pudesse desenhar a parte do objeto escondida. Após cada construção, era solicitada nova forma, perguntando-se à criança se ainda era possível que a parte escondida fosse de um outro jeito que não aquele imaginado.

Critérios de classificação: Nível IA - os sujeitos imaginam que a parte escondida só pode ser de um único jeito, idêntico ao da parte visível, podendo apenas ser menor ou mais fina. Nível IB - os sujeitos apresentam duas ou três alternativas para completar a parte escondida. Nível II - caracteriza-se pela possibilidade de construções mais numerosas, embora ainda limitadas, podendo-se identificar famílias de copossíveis. Nível III - neste nível os co-possíveis estendem-se ao ilimitado; todas as possibilidades são 
aceitas, desde que se encaixem à parte visivelmente observada.

Posições possíveis dos dados sobre suportes: foram utilizados dois suportes, uma base triangular e outra quadrada, e três dados iguais e com faces de cores diferentes. A criança trabalhava com um suporte de cada vez e era solicitada a colocar os dados sobre aquela base, do jeito que quisesse. Após cada execução era-lhe perguntado se havia outro jeito de colocar os dados e pedia-se a realização.

Critérios de classificação: Nível IA- aparecem pequenas variações, mas dentro de uma mesma família de possíveis. Nível IB - apresenta maiores variações e até duas famílias. Nível II - caracteriza-se por variações marcantes e presença de pelo menos três famílias de possíveis. Nível III - as formas para colocar os dados são infinitas.

Processo de intervenção: Os sujeitos foram submetidos a sessões de intervenção nos conteúdos de Formas Parcialmente Escondidas (FPE) e Eqüidistância (EQ), por intermédio da técnica do conflito cognitivo (Sisto,1993), sendo que um grupo recebeu intervenção em FPE; outro em EQ; o terceiro grupo em FPE e depois em EQ e o quarto em EQ e depois em FPE. As sessões foram individuais e duraram em média 30 minutos, encerrando-se na quarta sessão, mesmo que o sujeito não apresentasse as condutas pretendidas. Em cada sessão, os sujeitos foram colocados em 4 a 7 situações de conflito, dependendo da interação estabelecida e dos resultados dos conflitos observados. Todos os sujeitos foram póstestados, imediatamente ao final de cada intervenção e trinta dias após o primeiro, em três provas, quais sejam: formas parcialmente escondidas, equiidistância e posições possíveis dos dados, em seqüências aleatórias.

Sessões de Intervenção: foram padronizadas as questões colocadas aos sujeitos, podendo ocorrer eventuais modificações em virtude do encaminhamento dado pela criança à solução do problema. Eis exemplos de solicitações feitas para o conteúdo de equiidistância: "Coloque essas casas de um jeito que as crianças andem o mesmo tanto para chegar até a árvore. Você acha que desse jeito como você colocou as crianças andam o mesmo tanto? Como você faz para saber? Você acha que as casas podem ser arrumadas de outro jeito para as crianças andarem o mesmo tanto?". Exemplos de solicitações feitas para o conteúdo de formas parcialmente escondidas: "Eu tenho um objeto aqui que está metade escondido. Como você acha que é a parte que está escondida? Poderia ser de um outro jeito diferente desse que você fez?"
Procedimento para análise dos dados: para proceder à análise dos dados foram considerados a classificação dos sujeitos, o gênero e a idade. Neste último grupo classificaram-se como sujeitos mais novos os que se situavam no intervalo de $4 ; 6$ a $5 ; 8$ anos, e como mais velhos os que estavam no intervalo de $5 ; 9$ a $6 ; 9$ anos

\section{RESULTADOS}

As análises foram agrupadas em dois itens, em razão dos efeitos sobre a formação de possíveis no conteúdo não trabalhado na intervenção, ou seja, a posição dos dados. Primeiramente, são apresentadas as análises em relação aos efeitos produzidos pela intervenção por meio do conteúdo formas parcialmente escondidas e, em seguida, pela intervenção por meio do conteúdo eqüidistância.

\section{A. Efeito da intervenção em formas parcialmente escondidas na formação de possíveis em posição dos dados.}

A tabela 1 informa se o tipo de intervenção e a idade dos sujeitos produziram resultados estatisticamente diferentes e significativos, em relação ao desempenho atingido nos pós-testes 1 e 2 , para a prova das posições dos dados. Os resultados mostraram não haver efeito de interação significativo, como também as diferenças em relação ao gênero puderam ser atribuídas ao acaso.

Tabela 1. Valores de F (análise de variância) para as variáveis gênero, idade e tipo de intervenção nas situações experimentais (formas parcialmente escondidas, formas parcialmente escondidas/eqüidistância e eqüidistância/formas parcialmente escondidas), em relação ao desempenho na prova dos dados.

\begin{tabular}{lcccc}
\hline & \multicolumn{2}{c}{ Pós1-Dados } & \multicolumn{2}{c}{ Pós2-Dados } \\
\hline Tipo de intervenção & $\mathrm{F}=4,608^{* *}$ & \multicolumn{2}{c}{$\mathrm{F}=4,313^{* *}$} \\
Idade & \multicolumn{2}{c}{$\mathrm{F}=7,676^{* * * *}$} & $\mathrm{~F}=6,337 * *$ \\
Gênero & $\mathrm{F}=0,517$ & $\mathrm{~ns}$ & $\mathrm{~F}=0,003$ & $\mathrm{~ns}$ \\
Tipo de intervenção x idade & $\mathrm{F}=1,312$ & $\mathrm{~ns}$ & $\mathrm{~F}=0,113$ & $\mathrm{~ns}$ \\
Tipo de intervenção x Gênero & $\mathrm{F}=1,653$ & $\mathrm{~ns}$ & $\mathrm{~F}=0,077$ & $\mathrm{~ns}$ \\
Idade x Gênero & $\mathrm{F}=0,261$ & $\mathrm{~ns}$ & $\mathrm{~F}=0,333$ & $\mathrm{~ns}$ \\
Tipo de intervenção x Idade x Gênero $\mathrm{F}=0,261$ & $\mathrm{~ns}$ & $\mathrm{~F}=0,035$ & $\mathrm{~ns}$ \\
\hline
\end{tabular}

Legenda: Pós1 * $\mathrm{p}=0,006 \quad * * \mathrm{p}=0,016 \quad * * * \mathrm{p}=0,008 \quad$ Pós2 $\quad * \mathrm{p}=$ $0,017 * * \mathrm{p}=0,020 * * * \mathrm{p}=0,016$

Com relação à variável tipo de intervenção, verificou-se no pós-teste 1 sua interferência no desempenho dos sujeitos, produzindo médias cujas diferenças foram estatisticamente significativas $(\mathrm{F}=4,608$ e $\mathrm{p}=0,006)$. No pós-teste 2 mantém-se essa mesma tendência com valores de $\mathrm{F}=4,313$ e $\mathrm{p}=0,017$. 
Analisando as três situações experimentais, verificamos que o melhor desempenho na prova das posições possíveis de dados foi alcançado pelo grupo que passou por intervenção apenas no conteúdo (formas parcialmente escondidas), o qual obteve médias de desempenho muito semelhantes nos póstestes 1 e 2 (respectivamente de 2,63 e 2,75), encontrando-se pela prova $t$ de student valores de $\mathrm{t}=$ 0,808 e $p=0,432$. Dos grupos que passaram por intervenção em dois conteúdos, o que obteve o melhor desempenho na prova das posições possíveis de dados foi $o$ que teve intervenção na seqüência eqüidistância/formas parcialmente escondidas, o qual obteve médias iguais de desempenho $(2,22)$ nos dois pós-testes. O grupo com intervenção na seqüência formas parcialmente escondidas/eqüidistância obteve médias também muito semelhantes de desempenho de 2,00 (pós 1) e 2,10 (pós 2) - cuja diferença pode ser atribuída ao acaso $(\mathrm{t}=-0,698 \mathrm{e} \mathrm{p}=0,494)$.

A idade foi outra variável que isoladamente provocou diferenças estatisticamente significativas no conjunto das medidas. No pós-teste 1 , foram encontrados valores de $\mathrm{F}=7,676$ e p=0,008 e no pós-teste 2 , os valores de F e p foram respectivamente de 6,337 e 0,016.

Analisando-se a tendência de desempenho dos sujeitos, verificou-se que no pós-teste 1 os mais velhos obtiveram uma média de desempenho maior $(2,50)$ que a dos mais novos $(2,06)$, sendo essa mesma tendência verificada no pós-teste 2 , quando os sujeitos mais velhos atingiram a média de 2,58 e os mais novos, a de 2,13.

\section{B. Efeito da intervenção em eqüidistância na formação de possíveis em posições dos dados.}

Os dados da tabela 2 mostram que no pós-teste 1 a idade e $\mathrm{o}$ gênero foram as variáveis que apresentaram diferenças com significação estatística, ao contrário dos grupos formados pelos tipos de intervenção. Também não foi detectado nenhum efeito de interação.

Tabela 2. Valores de F (análise de variância) para as variáveis gênero, idade e tipo de intervenção nas situações experimentais (eqüidistância, formas parcialmente escondidas/eqüidistância e eqüidistância/formas parcialmente escondidas), em relação ao desempenho na prova dos dados.

\begin{tabular}{lcccc}
\hline & \multicolumn{2}{c}{ Pós1-Dados } & \multicolumn{2}{c}{ Pós2-Dados } \\
\hline Tipo de intervenção & $\mathrm{F}=1,170$ & $\mathrm{~ns}$ & $\mathrm{~F}=1,844$ & $\mathrm{~ns}$ \\
Idade & $\mathrm{F}=5,664 * *$ & $\mathrm{~F}=4,000$ & $\mathrm{~ns}$ \\
Gênero & $\mathrm{F}=4,687 * * *$ & $\mathrm{~F}=0,822$ & $\mathrm{~ns}$ \\
Tipo de intervenção x idade & $\mathrm{F}=1,114$ & $\mathrm{~ns}$ & $\mathrm{~F}=0,748$ & $\mathrm{~ns}$ \\
Tipo de intervenção x Gênero & $\mathrm{F}=0,366$ & $\mathrm{~ns}$ & $\mathrm{~F}=0,592$ & $\mathrm{~ns}$ \\
Idade x Gênero & $\mathrm{F}=0,144$ & $\mathrm{~ns}$ & $\mathrm{~F}=0,38$ & $\mathrm{~ns}$
\end{tabular}

\begin{tabular}{llccc} 
Tipo de intervenção $x$ Idade $x$ Gênero & $\mathrm{F}=0,219$ & $\mathrm{~ns}$ & $\mathrm{~F}=0,188 \quad$ ns \\
\hline Legenda: Pós 1 & $* \mathrm{p}=0,007$ & $* * \mathrm{p}=0,021$ & $* * * \mathrm{p}=0,036$
\end{tabular}

No pós-teste 1 , encontramos, para a variável idade, valores de $\mathrm{F}=5,664$ e $\mathrm{p}=0,021$, sendo que os sujeitos mais novos atingiram a média de 1,93 e os mais velhos, a de 2,46. A tendência geral observada, independentemente da situação experimental e do pósteste analisado, fosse ele imediato ou retardado, foi a de os sujeitos mais novos apresentarem um desempenho mais baixo que o dos mais velhos.

Com relação ao gênero, encontramos no pós-teste 1 um $\mathrm{F}=4,687$ e um $\mathrm{p}=0,036$, sendo que as meninas tiveram um desempenho superior $(2,50)$ ao alcançado pelos meninos (1,96), independente do tipo de intervenção a que foram submetidas.

Nos resultados do pós-teste 2, nenhuma das variáveis isoladamente, nem tampouco as interações entre tipo de intervenção, idade e gênero, provocaram significativas diferenças do ponto de vista estatístico, não tendo $\mathrm{o}$ valor de $p$ atingido o nível de significância de 0,05 .

\section{CONCLUSÕES}

As questões norteadoras deste estudo poderiam ser traduzidas como: qual a importância atribuída à maturação e ao gênero nas aprendizagens e sua transferência? Como os tipos de aprendizagem se relacionam? Como se dá a transferência em aprendizagem?

Pelos resultados encontrados nos experimentos pode-se afirmar que, na situação de conflito cognitivo em formas parcialmente escondidas, a idade e o gênero foram variáveis que interferiram no desempenho dos sujeitos. Do ponto de vista do desempenho no conteúdo posição dos dados, verificou-se que a situação experimental que trabalhou somente com o conteúdo formas parcialmente escondidas foi mais benéfica, seguida da seqüência de intervenção eqüidistâncialformas parcialmente escondidas e por último formas parcialmente escondidas/eqüidistância.

Verificou-se ainda que a idade dos sujeitos também interferiu na variação dos desempenhos em três das quatro situações analisadas, os mais velhos com melhor desempenho que os mais novos. A variável gênero teve efeito significativo somente no pós-teste 1 e para as situações experimentais que tiveram em comum o conteúdo de eqüidistância, tendo apontado para a superioridade de desempenho das meninas.

Três aspectos devem ser ressaltados nos resultados obtidos nos experimentos: 1) a maturação e 
o gênero parecem desempenhar um papel nas aprendizagens; 2) houve mudanças no desempenho dos sujeitos em relação a um conteúdo não ensinado, sugerindo a ocorrência de transferência; 3) houve superioridade de uma situação experimental sobre outra.

Com relação à variável idade, esse estudo confirmou a literatura, ou seja, o aspecto maturacional tem se destacado no desempenho dos sujeitos submetidos a aprendizagem. Assim, as crianças mais velhas apresentaram desempenho superior ao das mais novas. Esses achados reforçam a tese de Piaget de que não se pode acelerar indefinidamente o processo e que existe um tempo ótimo para que as mudanças ocorram de forma mais efetiva.

Quanto ao gênero, encontrou-se que as meninas apresentaram superioridade no desempenho nas situações de aprendizagem. No entanto a diferença entre gêneros é muito polêmica e os estudos são controversos, apontando a necessidade de mais averiguações.

A discussão que se segue pretende abordar os dois últimos aspectos colocados anteriormente. Assim, pode-se indagar sobre o "para que" servem as aprendizagens e "como" elas servem ao sistema cognitivo.

As interpretações teóricas feitas neste estudo partem do princípio de que todas as relações estabelecidas pelo ser humano, relativas ao seu funcionamento interno e ao meio externo, passam por um processo de regulação interna. Segundo Sisto (1997), ao buscar regulações entre o ambiente interno e o externo, esse processo estaria fazendo escolhas ou selecionando os elementos que poderiam restabelecer o equilíbrio interno e reconduzir o organismo a um estado mais adaptado. Em conseqüência, aceita-se que a aprendizagem não é um mero produto do meio exterior, em que os melhores e os piores comportamentos, ou respostas "certas" e "erradas", são mantidos ou eliminados em decorrência de seleções ambientais.

A teoria behaviorista defende a idéia de um processo generalizador em que a resposta aprendida e condicionada em uma dada situação poderia ser evocada, sem condicionamento, em uma nova situação, sendo que a força dessa evocação seria tanto maior quanto mais próximas fossem as características que as situações tivessem em comum (Keller, 1974). A "resposta por analogia", conforme se expressou Thorndike, reflete a possibilidade de a conduta do ser humano se repetir em situações similares.

Em que residiriam, então, as diferenças entre a proposta aqui aceita e as idéias básicas da teoria do reforço? Para Piaget (1985), o conhecimento também pode ocorrer por analogias, uma vez que o "novo" é encaixado por acomodações sucessivas aos conhecimentos anteriores (ao "velho"). No entanto, a essa tentativa de assimilar o "novo" valendo-se do "velho" se acrescenta o fato de que esse processo sempre comporta modificações que produzem um novo conhecimento, ou um conhecimento inexistente no sistema cognitivo do ser cognoscente. Assim, a diferença básica entre esses dois modelos teóricos reside no fato de que, para a teoria do reforço, os conhecimentos são generalizados a diferentes conteúdos ou contextos, mantendo as respostas muito próximas ou similares. Por sua vez, para a teoria construtivista, embora o processo tenha sido desencadeado por uma analogia, a resposta final não se mantém necessariamente próxima ou similar à que a gerou. Nesta perspectiva pode-se também pensar em analogia da forma, ou seja, em formas de procedimentos análogos, e não apenas em analogia de conteúdos.

Retomando a indagação do "para que" servem as aprendizagens observadas, pode-se dizer que as aprendizagens consolidadas estariam auxiliando as novas, não somente por seu grau de similaridade, mas porque estariam apoiando a construção de redes de ligações. Essas redes desencadeariam um processo de "construções mediadoras intrínsecas" (Sisto, 1997), pois ao se construir uma nova aprendizagem, o caminho percorrido para a construção serve de ponto de ligação para os conhecimentos que ainda estão por vir. Assim, nos experimentos verificou-se a ocorrência de mudanças no desempenho dos sujeitos em um conteúdo não trabalhado, de um pós-teste a outro e entre as situações experimentais, sugerindo que essas aprendizagens atuaram sobre esse outro conteúdo, não submetido a aprendizagem. Ou seja, houve transferência e de forma diferenciada. Mas que tipo de transferência seria essa, uma vez que os conteúdos e as respostas esperadas não se assemelhavam? Que tipos de ligações se estabeleceram?

Para Sisto (1997), a explicação parece se encontrar no próprio sistema, que funcionaria como canais abertos para as futuras aprendizagens, sem que se possa afirmar por qual deles se caminhará. Assim, o trabalho só com o conteúdo de formas parcialmente escondidas favoreceu aberturas na prova de posições dos dados, fazendo supor que prevaleceu o caminho das similaridades, embora similaridades de forma. São referentes à forma porque as respostas das situações colocadas na intervenção não foram ensinadas aos sujeitos, eles não recebiam feedback para suas respostas, e as respostas esperadas em ambos os 
conteúdos eram diferentes. Nesse contexto, a 'transferência' para a situação das posições dos dados teria o caráter de uma nova construção, e restaria, como explicação, apenas a transferência do mecanismo gerenciador da produção de analogias.

Restaria indagar sobre como reagiria o sistema cognitivo, quando submetido a várias aprendizagens num curto intervalo de tempo, como acontece na situação escolar. Os experimentos descritos demonstraram que algumas aprendizagens, seguidas de outras, favoreceriam mais pontos de contato e que estes possibilitariam a aprendizagem de outros conhecimentos.

Ao lado disso, observou-se que a seqüência de conteúdos trabalhada com os sujeitos, em situação de aprendizagem, teve uma forte interferência sobre a aprendizagem de um conteúdo não trabalhado com os sujeitos. Percebe-se nitidamente a superioridade do tipo de intervenção que trabalhou com a sequiência eqüidistância/formas parcialmente escondidas, embora os dois tipos de intervenção que trabalharam com seqüências invertidas tenham resultado em certa evolução na prova dos dados. Nesse sentido, em estudo anterior Martinelli (1999) e Sisto \& Martinelli (1998) já haviam demonstrado a influência que um conteúdo apresentado aos sujeitos em situação de intervenção por meio do conflito cognitivo teria sobre outro.

Assim é que as aprendizagens não ocorrem de forma isolada, nem parecem se restringir a uma situação específica, mas o processo continua em andamento, estendendo-se para situações novas. $\mathrm{O}$ tempo de elaboração dessas novas aprendizagens, bem como o caminho a ser seguido, parece estar sendo influenciado tanto pelas aprendizagens quanto pelas similaridades encontradas. Isso explicaria em parte as defasagens de conhecimentos que se têm, bem como as relações que cada sistema cognitivo estabelece entre os conhecimentos.

\section{BIBLIOGRAFIA}

Brison, D.W. (1966). Acceleration of conservation of substance. The Journal of Genetic Psychology, 109, 311-322.

Garcia, D.V. (1999). O conflito sócio-cognitivo como mediação entre maturação, gênero e aprendizagem. Tese de Doutorado, Universidade Estadual de Campinas, Campinas.

Gruen, G.E. (1965). Experiences affecting the development of number conservation in children. Child Development, $\underline{36}$, 963-979.

Keller, F.S. (1974). Aprendizagem: teoria do reforço. (R. Azzi, L. Zimmerman e L. O.de S. Queiroz.Trads.) São Paulo:EPU.

Liesenberg, M.T. (1994). Conflito cognitivo, possíveis e operatoriedade. Unimar, $\underline{16}, 59-81$.
Louro, J.R.O (1993). Aprendizagem cognitiva e multiplicação de procedimentos possíveis. Dissertação de Mestrado, Universidade Estadual de Campinas, Campinas.

Lutzer, V.D. (1987). Perceptual learning of a no-focal color discrimination between ages three and six. Journal of General Psychology, 114 (3), 273-279.

Martinelli, S.C. (1994). Aprendizagem da criatividade lógica possível ou não? Unimar, 16 , 41-57.

Martinelli, S.C. (1999). Sequiência de aprendizagem: um sistema de requisito? Em: Sisto, F.F. (Org). O cognitivo, o

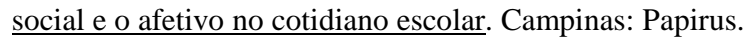

Morf, A (1959). Aprendizagem de uma estrutura lógica concreta (inclusão): efeitos e limites. Em: A Morf, J.Smedslund, A Vinh-Bang \& J.B. Wohlwill (Orgs). Aprendizagem das estruturas lógicas. Paris: PUF.

Padilla, M. \& Ollila, L. (1980). Effect of small-group teaching on acquisition and transfer of nonvisual seriation abilities. Science-Education, 64, 357-366.

Pavanello, R.M. (1995). Formação de possibilidades cognitivas em noções geométricas. Tese de Doutorado, Universidade Estadual de Campinas, Campinas.

Pereira, R.C.B. (1995). Conflito cognitivo, formação de possíveis e construção operatória. Trajetos, $\underline{6}, 100-117$.

Piaget, J. (1964). Development and learning. Journal of research in science teaching, $2,176-180$.

Piaget, J. (1985). O possível e o necessário: evolução dos possíveis na criança. (Vol.1) Porto Alegre: Artes Médicas.

Riding, R.J. \& Armstrong, J.M. (1982). Sex and personality differences in performance on matematics tests in 11-yearold. Children Educational Studies, $\underline{8}$ (3). 217-225.

Sisto, F.F. (1993). Fundamentos para uma aprendizagem construtivista. Pro-Posições, $\underline{4}$ (2), 38-52.

Sisto, F.F. \& Martinelli, S.C. (1998). Relações entre aprendizagens de diferentes conteúdos. Psico-USF, $\underline{3}$ (2), 39-57.

Sisto, F.F. (1997). Aprendizagem e mudanças cognitivas em crianças. Petrópolis: Vozes.

Sisto, F.F. (2000). Defasagem de desenvolvimento e transferência de aprendizagem construtivista. Psicologia: Reflexão e Crítica, 13 (1), 25-32.

Toh, K.A (1993). Gender and practical tasks in science. Educational Research, 35 (3), 255-265.

Waghorn, L \& Sullivan, E.V. (1970). The exploration of transition rules in conservation of quantity (substance) using film mediated modeling. Acta Psychologica, 32, 65-80.

Worthington, R. K.Typpo, M \& Worthington Jr., E.L. (1980). Spatial concept learning in preschool children: motoric experiences and verbal repetition as adjuncts to massive

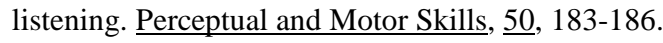

Yaegashi, S.F.R. (1994). Aprendizagem de possíveis e aquisições operatórias. $\underline{\text { Unimar, }}$ 16, 83-107.

Recebido em 20/04/2001

Revisado em 15/10/2001 Aceito em 25/10/2001 
